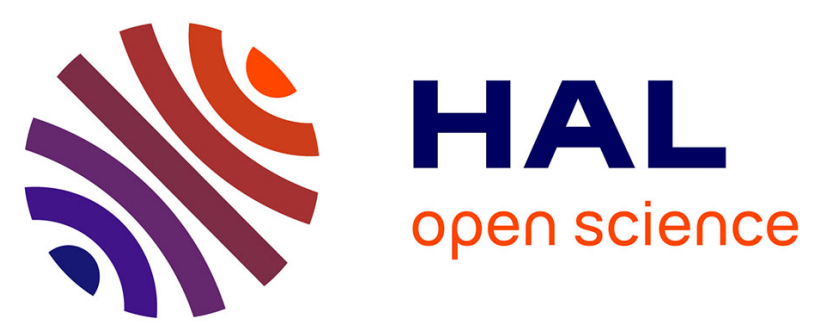

\title{
Sibling status, home birth, tattoos and stitches are risk factors for chronic hepatitis B virus infection in Senegalese children: A cross-sectional survey
} Lauren Périères, Camelia Protopopescu, Gora Lo, Fabienne Marcellin, El Hadji Ba, Marion Coste, Coumba Touré Kane, Aldiouma Diallo, Cheikh Sokhna, Sylvie Boyer, et al.

\section{To cite this version:}

Lauren Périères, Camelia Protopopescu, Gora Lo, Fabienne Marcellin, El Hadji Ba, et al.. Sibling status, home birth, tattoos and stitches are risk factors for chronic hepatitis B virus infection in Senegalese children: A cross-sectional survey. Journal of Viral Hepatitis, 2021, 28 (11), pp.1515-1525. 10.1111/jvh.13589 . hal-03355358

\section{HAL Id: hal-03355358 \\ https: / hal-amu.archives-ouvertes.fr/hal-03355358}

Submitted on 28 Jan 2022

HAL is a multi-disciplinary open access archive for the deposit and dissemination of scientific research documents, whether they are published or not. The documents may come from teaching and research institutions in France or abroad, or from public or private research centers.
L'archive ouverte pluridisciplinaire HAL, est destinée au dépôt et à la diffusion de documents scientifiques de niveau recherche, publiés ou non, émanant des établissements d'enseignement et de recherche français ou étrangers, des laboratoires publics ou privés. 


\title{
Sibling status, home birth, tattoos and stitches are risk factors for chronic hepatitis B virus infection in Senegalese children: A cross-sectional survey
}

\author{
Lauren Périères $^{1}$ (1) | Camelia Protopopescu ${ }^{2}$ (i) | Gora Lo $^{3}$ | Fabienne Marcellin ${ }^{2}$ () | \\ El Hadji Ba ${ }^{1}$ | Marion Coste ${ }^{2,4}$ ๑ | Coumba Touré Kane ${ }^{3}$ | Aldiouma Diallo ${ }^{1}$ ( ) | \\ Cheikh Sokhna $^{5}$ ( ) Sylvie Boyer ${ }^{2}$ @ | the ANRS 12356 AmBASS survey Study Group
}

\author{
${ }^{1}$ VITROME, Campus IRD-UCAD, Dakar, Senegal \\ ${ }^{2}$ Aix Marseille Univ, INSERM, IRD, SESSTIM, Sciences Économiques \& Sociales de la Santé \& Traitement de l'Information Médicale, ISSPAM, Marseille, France \\ ${ }^{3}$ Institut de Recherche en Santé de Surveillance Epidémiologique et de Formation, Dakar, Senegal \\ ${ }^{4}$ Aix Marseille Univ, CNRS, EHESS, Centrale Marseille, AMSE, Marseille, France \\ ${ }^{5}$ VITROME, IRD, AMU, AP-HM, SSA, IHU- MI, Marseille, France \\ Correspondence \\ Camelia Protopopescu, Aix Marseille University, INSERM, IRD, SESSTIM, Sciences Économiques \& Sociales de \\ la Santé \& Traitement de l'Information Médicale, ISSPAM, Marseille, France. Email: camelia.protopopescu@inserm.fr \\ Funding information \\ The AmBASS research project was funded in full by the French ANRS Emerging Infectious Diseases research agency under the auspices of the French National \\ Institute for Health and Medical Research (INSERM) (INSERM-ANRS), grant number 12356.
}

\begin{abstract}
Sub-Saharan Africa's hepatitis B virus (HBV) burden is primarily due to infection in in-fancy. However, data on chronic HBV infection prevalence and associated risk factors in children born post-HBV vaccination introduction are scarce. We estimated hepatitis B surface antigen (HBsAg) prevalence and risk factors in Senegalese children born dur-ing the HBV vaccination era. In 2018-2019, a community-based cross-sectional sur-vey was conducted in Senegal among children born between 2004 and 2015 (ie after the three- dose HBV vaccine series was introduced (2004) but before the birth dose's introduction (2016)). HBsAg-positive children were identified using dried blood spots. A standardized questionnaire collected socioeconomic information. Data were age- sex weighted and calibrated to be representative of children living in the study area. Risk factors associated with $\mathrm{HBsAg}$ positivity were identified using negative binomial

regression. Among 1,327 children, 17 were $\mathrm{HBsAg}$-positive (prevalence $=1.23 \%(95 \%$ confidence interval $[\mathrm{Cl}] \mathrm{0.61}-1.85)$ ). Older age (adjusted incidence-rate ratio [aIRR] 1.31 per one-year increase, 95\% Cl 1.10-1.57), home vs healthcare facility delivery (alRR 3.55, 95\% Cl 1.39-9.02), stitches (lifetime) (alRR 4.79; 95\% Cl 1.84-12.39), tat-toos (alRR 8.97, 95\% Cl 1.0179.11) and having an HBsAg-positive sibling with the same mother (aIRR 3.05, 95\% Cl 1.09-8.57) were all independently associated with $\mathrm{HBsAg}$ positivity. The low $\mathrm{HBsAg}$ prevalence highlights the success of the Senegalese HBV vaccination program. To further reduce HBV acquisition in children, high-risk groups, including pregnant women and siblings of HBsAg-positive individuals, must be screened. Vital HBV infection prevention measures include promoting delivery in healthcare facilities, and increasing awareness of prevention and control procedures.
\end{abstract}

K E Y WO R D S

child, hepatitis B, prevalence, risk factors, Senegal

Abbreviations: alRR, adjusted incidence-rate ratio; DBS, dried blood spots; EPI, expanded program on immunization; GHSS, Global Health Sector Strategy on viral hepatitis; HBsAg, hepatitis B surface antigen; HBV, hepatitis B virus; HDSS, health and demographic surveillance system; SSA, sub-Saharan Africa. 


\section{1 | INTRODUCTION}

The expansion of routine hepatitis B virus (HBV) vaccination has led to a significant reduction in chronic HBV infection prevalence in children worldwide, from $4.7 \%$ among under-five-year-olds in the pre-vaccination era to $1.3 \%$ in $2015 .{ }^{1}$ However, HBV infection prevalence in children remains high in Africa, particularly in West Africa (estimated at $5.8 \%$ in five-year-olds in 2016). ${ }^{2}$

HBV is transmitted through mucous membrane or non-intact skin exposure to infected blood or other bodily fluids such as saliva, semen and vaginal fluid. ${ }^{3}$ In sub-Saharan Africa (SSA), infection most commonly occurs in early childhood through horizontal transmission (close interaction with infected household contacts and playmates) and mother-to-child transmission during delivery. ${ }^{4-9}$ Other infection pathways include poor infection control procedures during surgery ${ }^{10}$ and traditional practices (eg tattooing). ${ }^{10,11}$

The risk of developing chronic HBV infection is inversely related to age at infection, occurring in up to $90 \%$ of those infected perinatally, but falling to less than $5 \%$ in individuals infected during adulthood. ${ }^{4}$ Although HBV infection may remain asymptomatic and even resolve spontaneously, in up to $50 \%$ of cases, chronic HBV infection can lead to liver disease and related complications, and even death. ${ }^{12}$

The prevalence of hepatitis $B$ surface antigen ( $\mathrm{HBsAg}$ ) in the general population reflects the endemicity of HBV infection. ${ }^{13}$ In Senegal, HBsAg prevalence in the general population is estimated at 8.1\% (95\% confidence interval [Cl], 7.5-9.0\%). ${ }^{2}$ Several control measures have been implemented in recent years, including the threedose series vaccine (administered 6, 10 and 14 weeks after birth) and the birth dose, introduced in the national Expanded Program on Immunization (EPI) in 2004 and 2016, respectively.

Estimating $\mathrm{HBsAg}$ prevalence in children and identifying associated risk factors is particularly important to better control the hepatitis B epidemic. Infection during early childhood is also associated with a higher risk of developing cirrhosis and hepatocellular carcinoma. ${ }^{14}$ However, to our knowledge, few studies to date have measured the prevalence of $\mathrm{HBsAg}^{11,15-18}$ and identified associated factors $^{15,17,18}$ in children born after the introduction of the HBV vaccine in SSA, including Senegal. This study aimed to (i) estimate the prevalence of chronic HBV infection in children born post-HBV vaccine introduction in a rural area of Senegal, and (ii) identify associated risk factors.

\section{2 | METHODS}

\section{1 | Study setting and design: the ANRS 12356 AmBASS survey}

The ANRS 12356 AmBASS cross-sectional survey aimed to assess the health and socioeconomic burden of chronic HBV infection at the individual, household and population levels in a rural area of Senegal. ${ }^{19}$ Data were collected from October 2018 to May 2019 in the Niakhar Health and Demographic Surveillance System (HDSS) facility, which is located 135 kilometres east of Dakar. The HDSS monitors demographic and health characteristics of adults and children living in 30 villages around Niakhar through regular data collection. Information on births, deaths, migrations and pregnancies is recorded in the HDSS database. ${ }^{20}$ In 2018, the HDSS covered a population of 44,854 individuals. ${ }^{20}$ Health care is provided by four healthcare posts managed by nurses in the HDSS area, as well as two healthcare centres managed by physicians (district level reference facilities) and a regional hospital, all located close to the HDSS area. ${ }^{19}$ Healthcare posts offer primary care including maternal and child health care, vaccination and child deliveries. Key actors at the community level include community health workers (who are responsible for health points, Senegal's lowest care level after healthcare posts, where health education and first aid care are provided) and community health volunteers (who promote maternal and child health).

The survey sample size was defined to provide an estimation of chronic HBV infection prevalence with at least $3 \%$ precision in the main age groups ( $\leq 14,15-34$ and $\geq 35$ years old). ${ }^{19}$ Two-stage stratified sampling of participating households was conducted. First, the villages covered by the HDSS were categorized into three semi-urban and 27 rural villages. The three semi-urban villages and eight randomly chosen rural villages were selected for participation. Second, 401 households were randomly selected from these 11 villages. All residents aged at least six months old in these households were invited to participate in the survey. For children, a parent or legal guardian had to be present at the time of the survey and had to provide consent for the child's participation.

The ANRS 12356 AmBASS survey methodology and sampling procedure have been previously described elsewhere. ${ }^{19}$

\subsection{Data collection for children}

All participating children were screened for HBV infection, and socioeconomic information was collected using a standardized questionnaire.

\subsection{1 | Serological assay}

Nurses performed home-based HBV screening using dried blood spots (DBS) on a Whatman 903 protein card saver. DBS have a high (>90\%) diagnostic sensitivity and specificity compared with plasma or serum. ${ }^{13,21}$ Using a standardized method, ${ }^{22}$ DBS were eluted to detect $\mathrm{HBsAg}$ by a chemiluminescent microparticle immunoassay (ARCHITECT, Abbott). To determine optimal cut-off values for positivity and negativity using DBS, we obtained paired capillary blood for DBS and venous blood for serum samples from 39 individuals in the pilot study of AmBASS, three of whom were HBsAg positive using a serum sample (see Appendix S1). We then determined $\mathrm{HBsAg}$ cut-off values of $1.0 \mathrm{IU} / \mathrm{ml}$ (for negativity) and $1.5 \mathrm{IU} / \mathrm{ml}$ (for positivity). For HBsAg levels between 1.0 and $1.5 \mathrm{IU} / \mathrm{ml}$ using DBS, 
we systematically performed a second HBsAg test using serum samples to confirm the status.

Once air-dried, DBS were frozen at $-20^{\circ} \mathrm{C}$ and transferred weekly for analysis to the Institute for Health Research, Epidemiological Surveillance and Training in Diamniadio, approximately $120 \mathrm{~km}$ from Niakhar. HBsAg was measured using chemiluminescence microparticle immunoassay (Abbott Diagnostics). ${ }^{19}$ Participants received their HBsAg screening results within one to two months.

\subsection{2 | Socioeconomic questionnaire}

A socioeconomic questionnaire was completed by each child's parent or legal guardian to document the child's socio-demographic characteristics, health history (impairments, hospitalization and illnesses), $\mathrm{HBV}$ vaccination status and exposure to HBV transmission risk factors. Vaccination history was determined from three data sources (i) children's vaccination cards (if available), (ii) six-monthly vaccination data from the HDSS database (based on vaccination cards) and (iii) vaccination records in healthcare posts (where possible).

Data were recorded electronically on tablets using Voxco Survey Software (version 2).

\subsection{Ethical considerations}

The study received ethical approval from the Senegalese National Ethical Committee for Research in Health (no. 082MSAS/DPRS/ CNERS), and authorization from the French Commission on Information Technology and Liberties (reference MMS/HG/OTB/ AR181521). The study conforms to the declaration of Helsinki.

\section{4 | Study population}

The study population of the present analysis included children born between 01 January 2004 (ie after the introduction of the threedose HBV vaccine series in Senegal's EPI) and 31 December 2015 (ie before the introduction of the birth dose in the EPI). Children born on or after 01 January 2016 were excluded from the analysis in order to have a homogeneous population with the same exposure to vaccination.

\section{5 | Study outcome}

The study outcome was chronic HBV infection. As per WHO recommendations for settings where $\mathrm{HBsAg}$ seroprevalence is above $0.4 \%$, we considered that testing positive for $\mathrm{HBsAg}$ at a single assessment (ie without subsequent confirmatory testing) represented chronic HBV infection. ${ }^{13}$

\subsection{Explanatory variables}

The following explanatory variables were tested as risk factors for chronic HBV infection:

- Socio-demographic characteristics: sex; age (years); enrolment in the Senegalese education system (yes/no/too young [ $<7$ years old]); birth order $(1 / 2 / \geq 3)$; type of village (semi-urban/ rural); household distance from the nearest healthcare post $(\leq 3 \mathrm{~km} />3 \mathrm{~km})$ and household living conditions index $\left(1^{\text {st }}\right.$ or $2^{\text {nd }} / 3^{\text {rd }}$ or $4^{\text {th }}$ quartile), built using a multiple component analysis of information on durable goods, agricultural and farming resources, and housing characteristics at the household level.

- Pentavalent vaccination status (complete/incomplete or delayed/missing information): 'complete' vaccination was defined as receiving all three doses of the pentavalent vaccine within the WHO-recommended timeframe (ie first dose received at least six weeks after birth and a delay of at least four weeks between each subsequent dose).

- HBV transmission risk factors: place of birth (healthcare facility (ie healthcare post, healthcare centre, hospital) or home); birth by caesarean section; previous hospitalization; lifetime stitches; invasive medical procedures (dental care, intravenous therapy, care involving injections or needles, care involving spring-loaded syringes); scarification; tattoos; piercings; sharing of personal hygiene items (toothbrush, razor, hair needle, manicure or pedicure equipment); consumption of pre-chewed food; and circumcision.

- HBV status of other compound (ie the 'residential unit' in the HDSS database, and understood as a grouping of one or several households ${ }^{20}$ ), household or family members: at least one HBsAgpositive individual (adult or child) in the compound; at least one $\mathrm{HBsAg-positive} \mathrm{child} \mathrm{in} \mathrm{the} \mathrm{compound;} \mathrm{at} \mathrm{least} \mathrm{one} \mathrm{HBsAg-}$ positive individual in the household; at least one $\mathrm{HBsAg}$-positive child in the household; at least one HBsAg-positive sibling (same mother); at least one HBsAg-positive sibling (same father); mother's HBV status.

\subsection{Statistical analyses}

\subsection{1 | Data weighting and calibration}

Data were weighted and calibrated to ensure that the study sample was representative of the population living in the Niakhar HDSS in terms of sex and age. To do this, sampling weights were calculated as the inverse of the individual probability of inclusion in the sample. Final weights were obtained after multiplying sampling weights by calibration factors, calculated as the ratio of the percentage of individuals in the HDSS demographic database to the percentage of individuals in the survey sample for each age and sex group. ${ }^{19}$ Weighted and calibrated data were used for all analyses. 


\subsubsection{Chronic HBV infection prevalence estimation}

The prevalence of chronic HBV infection was calculated as the percentage of $\mathrm{HBsAg}$-positive individuals among those with available and valid biological data. The $95 \% \mathrm{Cl}$ was estimated using the standard Wald $\mathrm{Cl}$ for proportions.

\subsection{3 | Descriptive analyses}

The study population's socio-demographic characteristics and exposure to HBV risk factors were described using percentages for categorical variables and means \pm standard deviations for continuous variables.

\subsection{4 | Regression model}

Factors associated with chronic HBV infection were identified using a negative binomial regression model. The choice of this model was justified by the low number of $\mathrm{HBsAg}$-positive children. ${ }^{23}$ Variables with a $p$ value $<.25$ (Wald chi-square test) in the univariable analysis were considered eligible to enter the multivariable model. Backward stepwise selection was used to determine variables in the final multivariable model with a $p$ value threshold set at 0.05 .

We also conducted a sensitivity analysis in the population of children born between 2004 and 2018 (ie including children born after the introduction of the birth dose in 2016).

All statistical analyses were performed using Stata, version 14.2 for Windows (StataCorp).

\section{3 | RESULTS}

\subsection{Chronic HBV infection prevalence}

Among the 1,327 children in the study population, 17 had chronic HBV infection; prevalence was $1.28 \%(95 \% \mathrm{Cl}, 0.68-1.89)$ using crude data, and $1.23 \%(95 \% \mathrm{Cl}, 0.61-1.85)$ using weighted and calibrated data. Figure 1 shows the distribution of HBsAg positivity per birth year.

\section{2 | Socio-demographic characteristics of study participants}

Mean age was $8.80 \pm 3.83$ years. Approximately half were male (52.48\%) and two-thirds were born in healthcare facilities (62.23\%). The majority lived in semi-urban villages (61.30\%) located within three kilometres of the nearest healthcare post (72.33\%). HBV vaccination data were available for $63.97 \%$ of the sample. Of these, $49.88 \%$ had received all three doses of pentavalent vaccine within the recommended timeframe. Table 1 presents the socio-demographic characteristics of the whole study population and according to HBV status. Vaccination status, HBV status of siblings, HBV status of household or compound members and mother's HBV status for all 17 children with chronic HBV infection are presented in Appendix S2.

\section{3 | Prevalence of HBV transmission risk factors}

Some HBV infection risk factors were frequent in our study sample (Table 1). For instance, a high proportion of children shared personal hygiene items (40.19\%), had undergone invasive medical procedures (64.13\%) and lived in a compound (52.98\%) or individual household (48.74\%) with at least one HBsAg-positive individual. In contrast, stitches (lifetime) (6.36\%), scarification (4.25\%), tattoos (0.17\%) and a caesarean section at birth $(0.85 \%)$ were quite rare. Moreover, few participants lived in a compound (8.44\%) or household (6.44\%) where there was an HBsAg-positive child, and few had an HBsAgpositive sibling from the same mother (10.44\%) or father (11.52\%).

\subsection{Chronic HBV infection risk factors}

As none of the 17 children with chronic HBV infection were born by caesarean section, had been hospitalized in the past or consumed

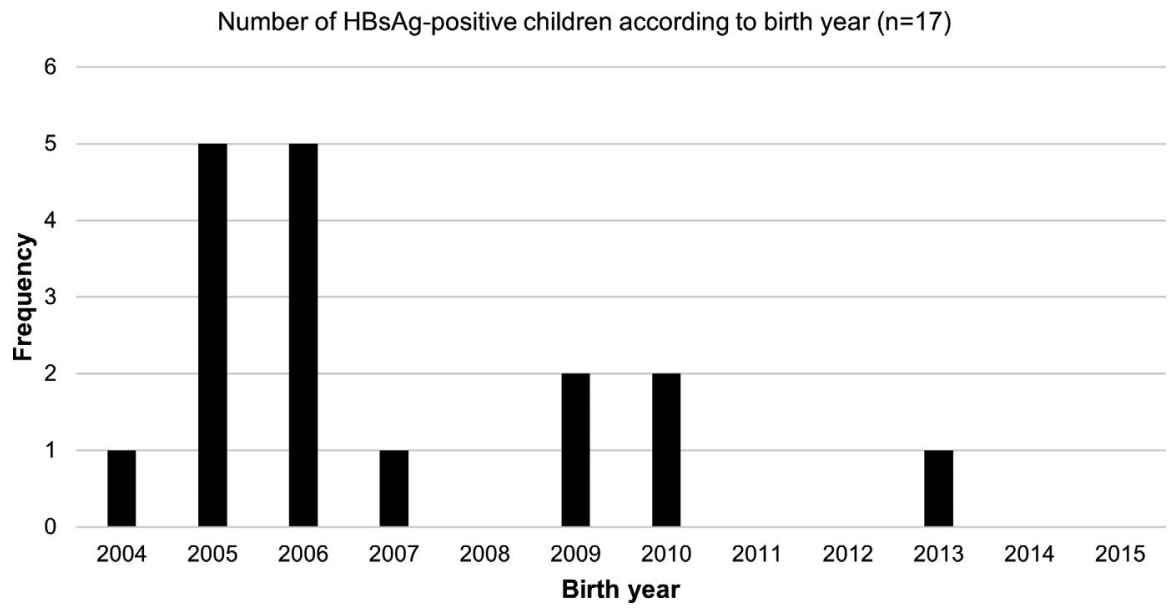

FIGURE 1 Number of HBsAg-positive children according to year of birth (ANRS 12356 AmBASS). HBsAg: hepatitis B surface antigen 


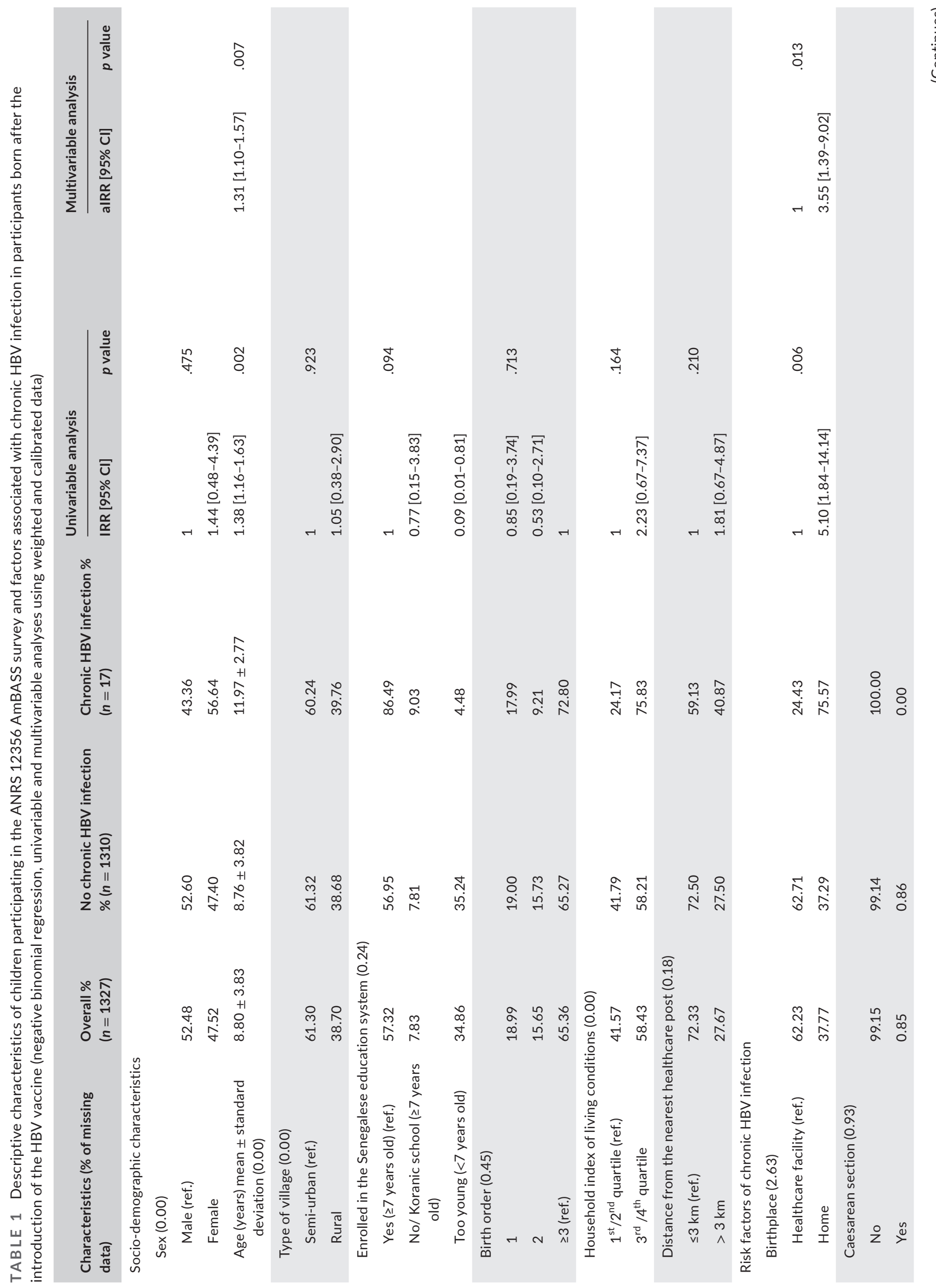



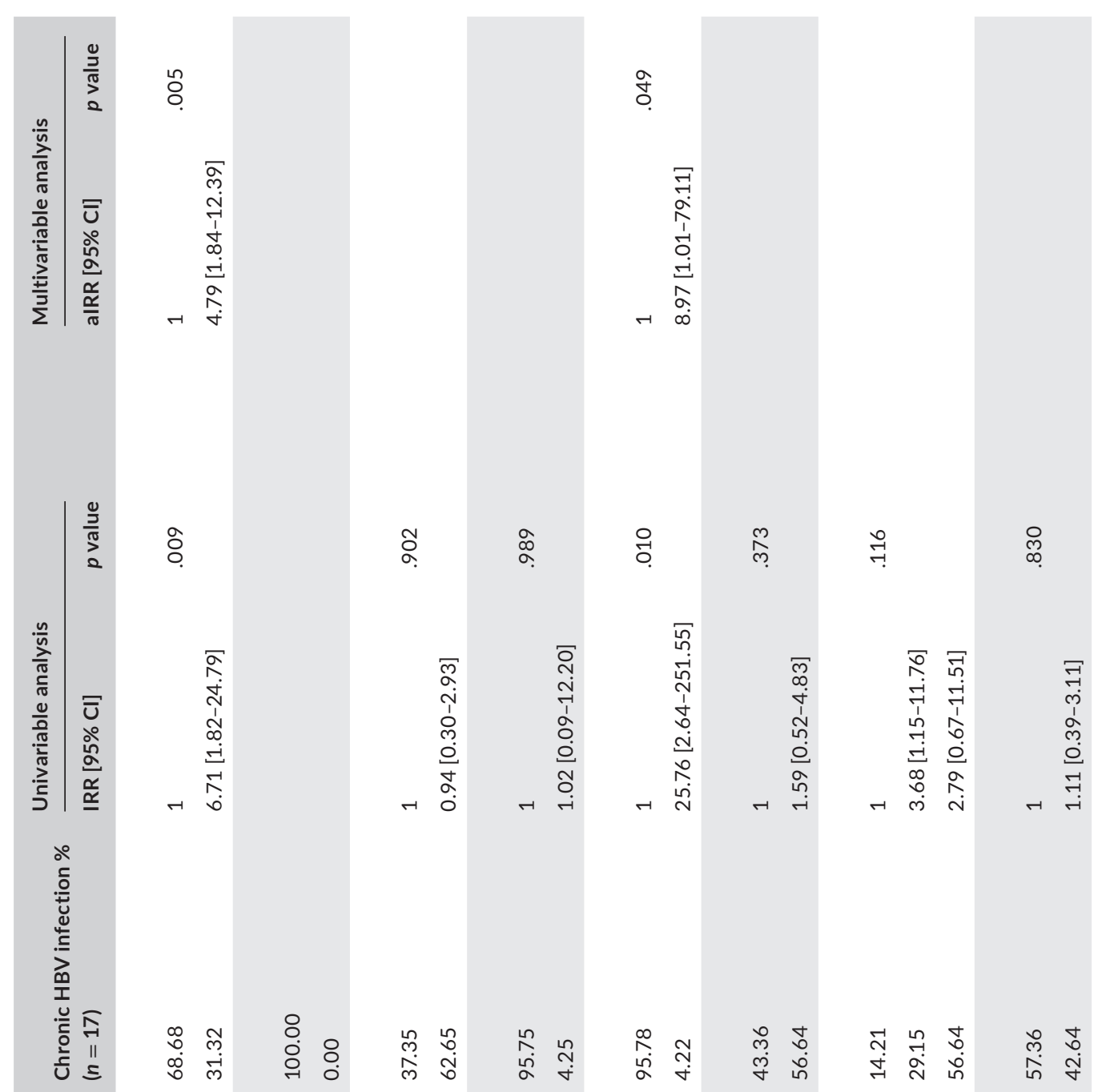

$\stackrel{n}{m}$ ก
$\stackrel{m}{m}$

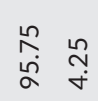

ฝุ กั

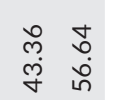

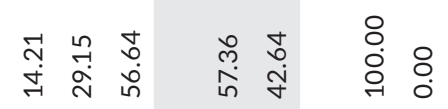

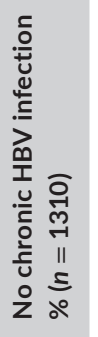

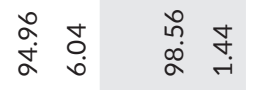

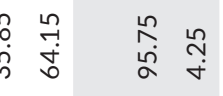

$\begin{array}{ll}\infty & \\ \infty & 7 \\ \alpha & \ddots \\ 0\end{array}$

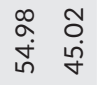

\begin{tabular}{lll}
0 & \multirow{2}{n}{} & $\hat{N}$ \\
$\ddot{m}$ & $\infty$ & \multirow{1}{*}{}
\end{tabular}

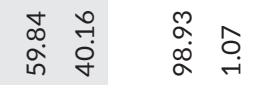

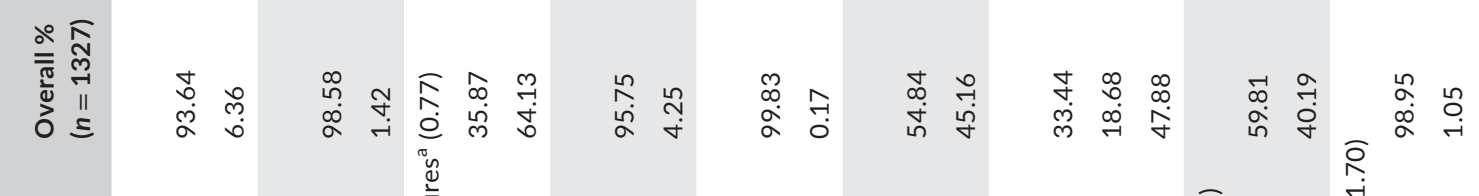

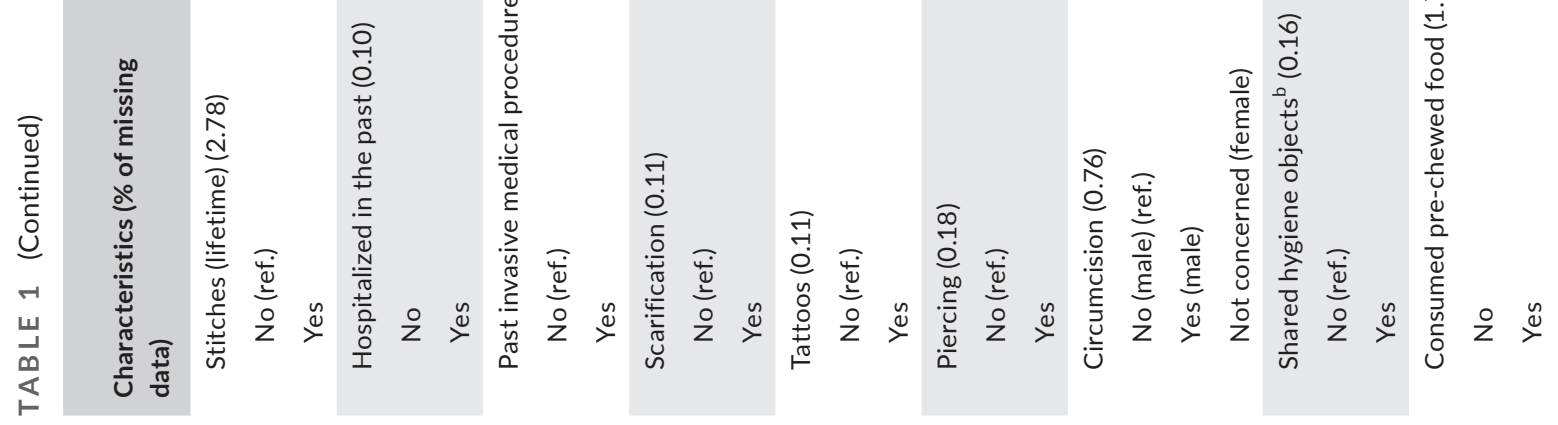




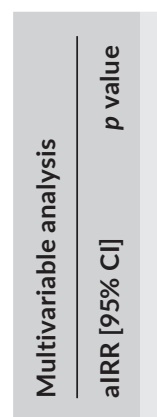

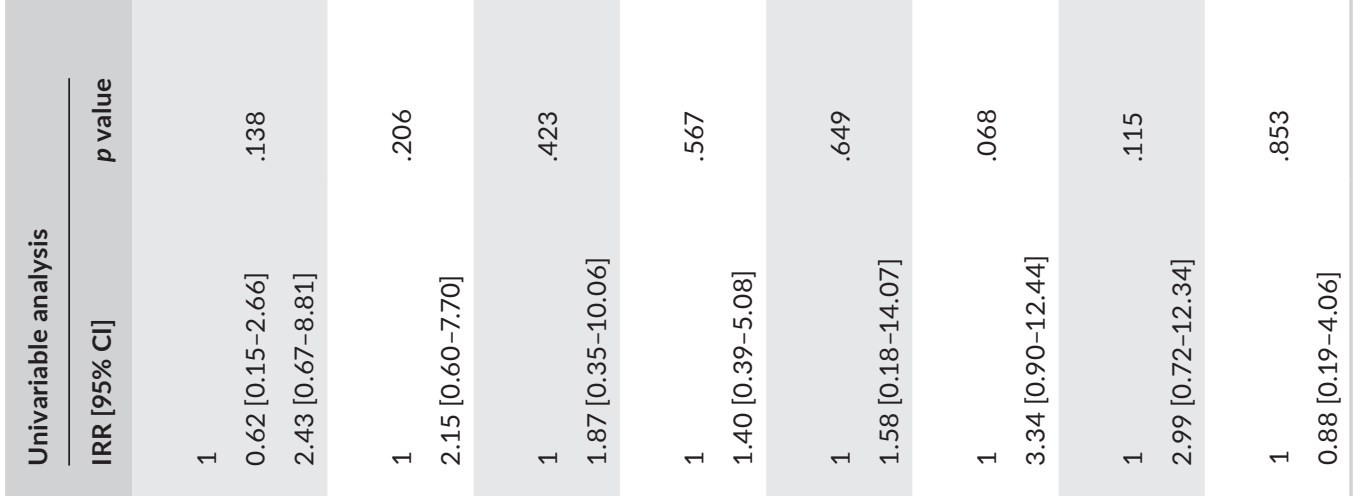
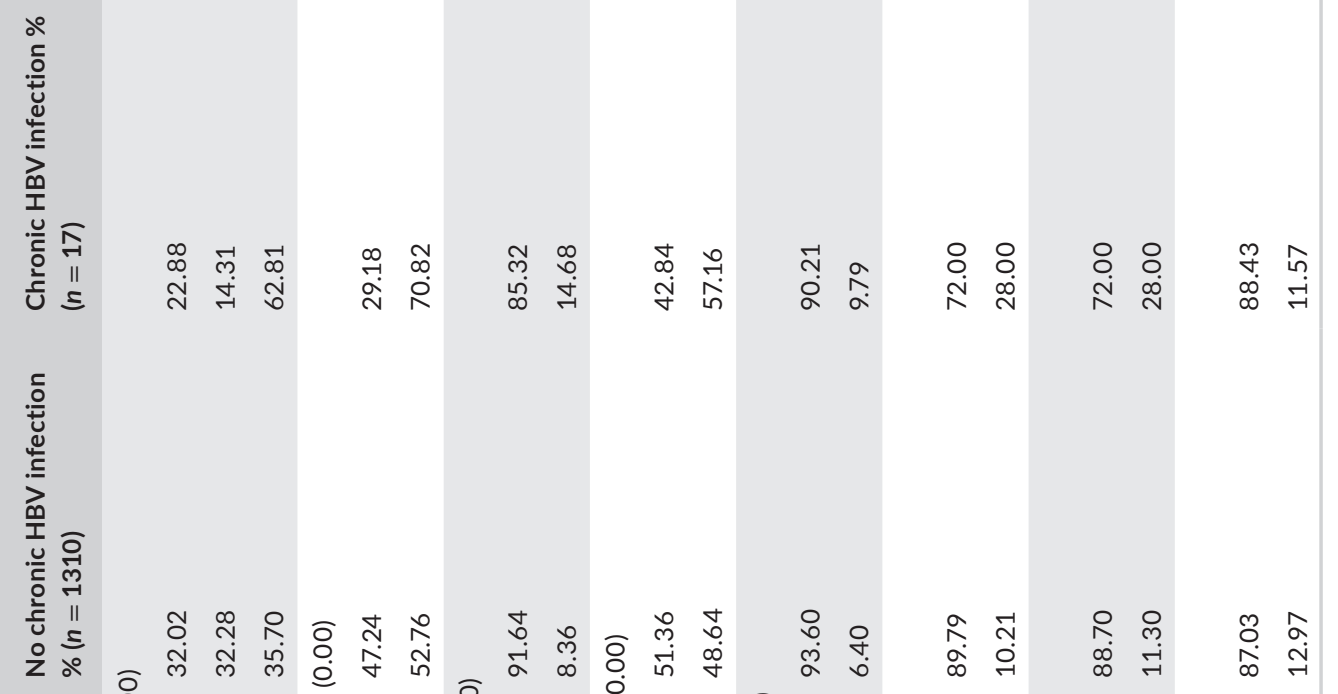

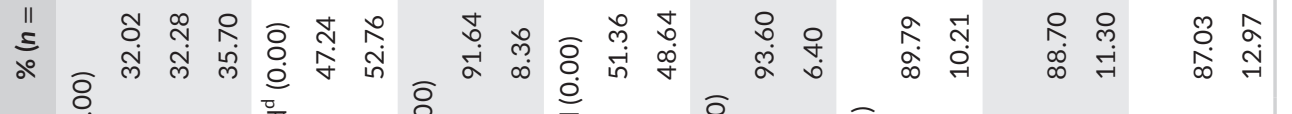

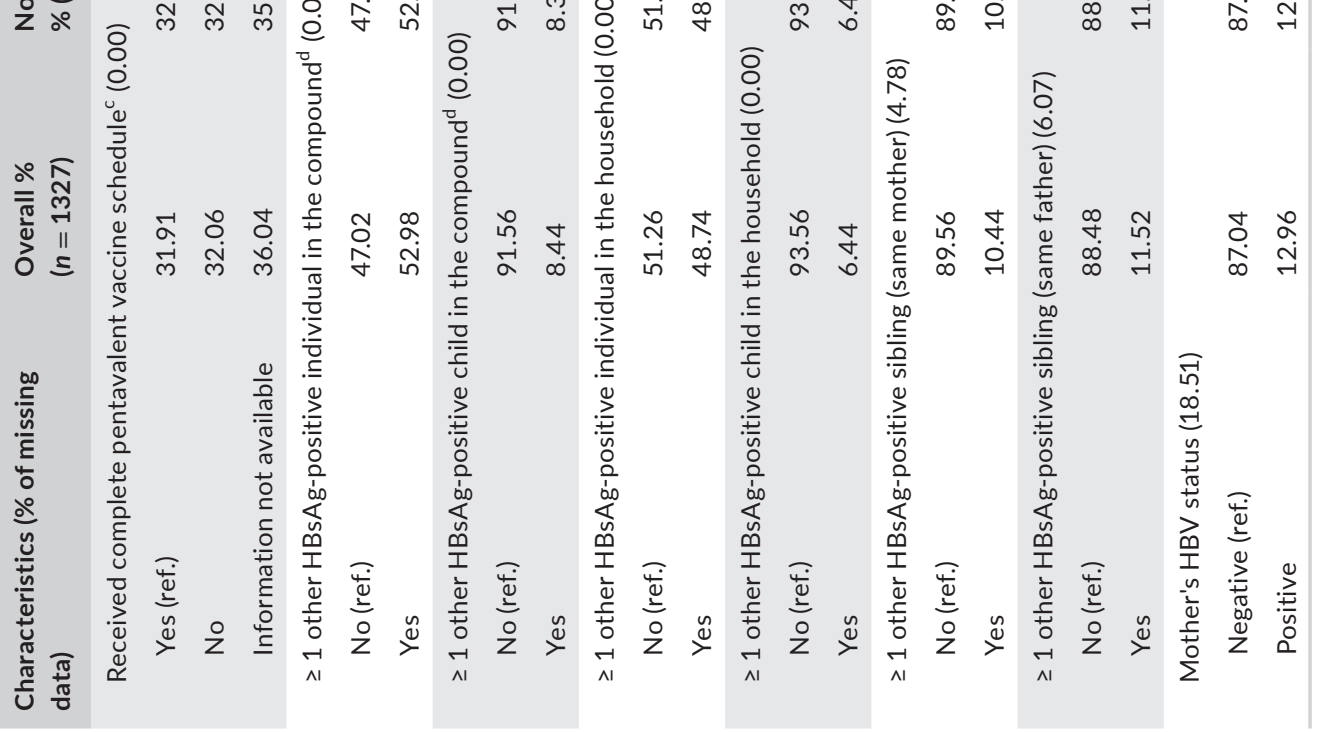

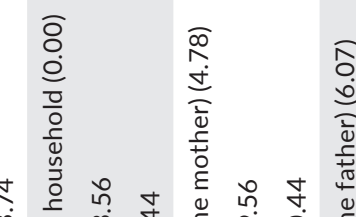


pre-chewed food, these variables were excluded from the regression analyses.

Factors associated with chronic HBV infection in univariable and multivariable analyses are presented in Table 1 . The following variables were eligible to enter the multivariable model ( $p$ value $<.25$ in univariable analysis): age, enrolment in the Senegalese education system, household index of living conditions, distance from the nearest healthcare post, place of birth, stitches, tattoos, circumcision, pentavalent vaccine schedule status, HBV status of other children in the compound, siblings' (same mother) HBV status and siblings' (same father) HBV status.

After multivariable adjustment, older age (adjusted incidencerate ratio [aIRR] of 1.31 per one-year increase, 95\% Cl 1.10-1.57), home birth (versus a healthcare facility) (aIRR 3.55, 95\% Cl 1.399.02), stitches (aIRR 4.79; 95\% Cl 1.84-12.39), tattoos (aIRR 8.97, 95\% Cl 1.01-79.11) and having an HBsAg-positive sibling (same mother) (aIRR 3.05, 95\% Cl 1.09-8.57) were all associated with HBsAg positivity.

The sensitivity analysis results were similar (Appendix S3).

\section{4 | DISCUSSION}

This is one of the few studies to date in SSA to both estimate the prevalence of chronic HBV infection and identify associated risk factors in children born after the introduction of the HBV vaccine. It is also the first to do so in Senegal using a representative sample of children living in a rural area. Our results show a very low prevalence of chronic HBV infection in children born after the vaccine's introduction. Older age, tattoos, stitches (lifetime), having a sibling (same mother) with $\mathrm{CHB}$, and home birth (versus healthcare facility) were all associated with chronic HBV infection.

The low prevalence of chronic HBV infection in children living in the Niakhar HDSS can, for the most part, be attributed to the introduction of the HBV vaccine in the national EPI in 2004, despite vaccination coverage variability in our study population. $\mathrm{HBsAg}$ prevalence in our study population reflected that found in hospitalized children in Dakar born after $2004(0.2 \% \text { and } 1.1 \%)^{15,16}$ and was slightly below the value estimated in a literature review and modelling study among five-year-old children in 2016 (1.6\%). ${ }^{2}$ This low prevalence in children strongly contrasts with the pre-vaccination $\mathrm{era}^{24}$ and with the prevalence estimated for individuals born before 2004 in the Niakhar HDSS (L. Périères, A. Diallo, F. Marcellin, M.L. Nishimwe, E. H. Ba, M. Coste, G. Lo, P. Halfon, C. Touré Kane, G. Maradan, P. Carrieri, A. Diouf, Y. Shimakawa, C. Sokhna, S. Boyer). Furthermore, Senegalese children have one of the lowest $\mathrm{HBsAg}$ prevalence in SSA since the vaccine's introduction in the region. ${ }^{11,17,18}$

Several risk factors associated with $\mathrm{HBsAg}$ positivity in our study population are consistent with findings from other studies on children in SSA. For instance, in Burkina Faso, children 5-10 years old had a higher risk of HBV exposure ( $\mathrm{HBsAg}$ or antibody to hepatitis $\mathrm{B}$ core antigen positivity, adjusted odds ratio $2.1,95 \% \mathrm{Cl} 1.2-3.8 \%$ ) than children $1-4$ years old. ${ }^{11}$ In our study, older age was associated with chronic HBV infection and the number of children who acquired HBV infection was highest in the years 2005 and 2006. This may be because children born shortly after the introduction of the HBV vaccine in the EPI had lower vaccination coverage than those born more recently. ${ }^{11}$ Although we found no association between vaccination status and $\mathrm{HBsAg}$ positivity, vaccination data were unavailable for $35.9 \%$ of the whole study population, and $62.8 \%$ of those with chronic HBV infection, particularly older children. HBV infection in older children may also be due to poor immune response to the vaccine in children born shortly after 2004. Indeed, a study conducted in 2010 in Dakar found that children born between 2006 and 2009 had a low immune response to the pentavalent vaccine, probably due to poor vaccine quality. ${ }^{25}$

In Nigeria, two studies found that tattoos were associated with $\mathrm{HBV}$ infection in children, although neither study performed multivariable analyses. ${ }^{10,18}$ Unsterilized needles for tattooing constitute a HBV transmission route through potential bleeding or ulceration. ${ }^{10}$

Few recent studies have tested all members of the same household for chronic HBV infection and assessed the risk of horizontal HBV transmission. The participation rate in the AmBASS survey was very high (91.5\%), suggesting that almost all household members were tested. Interestingly, having an HBsAg-positive sibling with the same mother was associated with $\mathrm{HBsAg}$ positivity in our study sample, while living in the same household or compound as an HBsAg-positive individual and having an HBsAg-positive sibling with the same father were not. In our study setting, children with the same mother were usually raised together, suggesting that sibling-to-sibling transmission is a common mode of HBV transmission in this context. This hypothesis reflects another recent study conducted in The Gambia which found that the risk of the youngest child in the household being HBsAg positive was related to the number of HBsAg-positive siblings. ${ }^{26}$ In contrast, chronic HBV infection was not associated with the mother's HBV status or with the child's birth order in our study, suggesting a low risk of mother-tochild transmission in our study setting. However, data on mother's HBV status was missing for $18.5 \%$ of all the study population and 2/17 of HBsAg-positive children. Furthermore, descriptive data suggest that mother-to-child transmission may have occurred in 2/17 HBsAg-positive children who had a mother with a positive HBV status despite the fact the former received three doses of pentavalent vaccine.

In the literature on chronic HBV infection in children in SSA, neither stitches nor home delivery is identified associated factors, despite both being known risk factors for HBV transmission. For example, in Ethiopia and Burkina Faso, home delivery was associated with past HBV exposure and lower immunity in children but not with $\mathrm{HBsAg}$ positivity, ${ }^{11,17}$ suggesting that past exposure to the virus could be due to the use of inadequately sterilized material during delivery. ${ }^{17}$ This may explain the association between home delivery and HBsAg positivity in children in our study. More specifically, the 12/17 HBsAg-positive children born at home were delivered by friends and family, not by traditional birth attendants, and it is likely that adherence to infection control procedures was insufficient. 
Moreover, mothers who gave birth at home may have been less likely to visit health centres or to vaccinate their child, leading to lower vaccine coverage. ${ }^{11}$ This hypothesis could not be confirmed in our study, probably because vaccination data were missing for a third of the sample. However, in another study conducted in the Niakhar HDSS among children born after 2016 (ie year of birth dose introduction in Senegal), home birth was associated with non-adherence to the complete hepatitis $B$ vaccination schedule (ie birth dose followed by at least two pentavalent doses). ${ }^{27}$

In 2016, the World Health Assembly adopted the 'Global health sector strategy on viral hepatitis' (GHSS), which aims to eliminate hepatitis $B$ as a public health problem by 2030 . To do so, a $90 \%$ reduction in $\mathrm{HBV}$ infection incidence (ie $0.1 \%$ prevalence of $\mathrm{HBsAg}$ in under-five-year-olds) and a $65 \%$ reduction in HBV mortality need to be achieved by $2030 .{ }^{5}$ Our study is particularly relevant, as locallevel epidemiological data are needed to provide information not only on the HBV epidemic and driving factors for transmission, but also on the progress being made in achieving the GHSS elimination goal. ${ }^{28}$

Our results have several implications for the hepatitis B elimination agenda in Senegal. First, they highlight the success of the country's hepatitis B vaccination program in reducing chronic HBV infection prevalence in children to close to $1 \%$ (ie GHSS target for 2020). ${ }^{5}$ The introduction of the birth dose in 2016 together with further improvements in vaccination coverage will continue to prevent new HBV infections and have already put Senegal on course to achieving the required $90 \%$ reduction in chronic HBV infection incidence to meet the 2030 GHSS target. ${ }^{5,27}$ This contrasts with many other countries worldwide which will not achieve this target in time because of insufficient investment in HBV elimination, including in vaccination. ${ }^{28}$

Second, free HBsAg screening should be systematically offered to women during the first trimester of pregnancy ${ }^{4}$ to encourage HBsAg-positive women to deliver in healthcare facilities and to ensure that their newborn receives the birth dose as soon as possible. Furthermore, as early diagnosis of HBV infection is key to prevent liver damage, HBV screening should be offered to all siblings as soon as their mother or other household member tests HBsAg positive. Currently, most individuals with chronic HBV infection worldwide are either undiagnosed or present with advanced/late stage liver disease which can lead to death. ${ }^{29}$ To develop HBV screening, free rapid diagnostic tests should be made available at all levels of care. $^{4,28}$

Third, training of healthcare professionals and community members on infection prevention and control procedures should be reinforced to prevent HBV transmission during tattooing, stitching and home delivery.

Our study has several limitations. First, its cross-sectional design and the lack of phylogenetic data because of insufficient spots left on DBS, limited our ability to precisely establish the types of HBV transmission. Second, the low number of HBsAg-positive children in the sample meant that estimates for some factors were less precise, with large Cls of alRRs (eg for the 'tattoos' risk factor). Third, there may have been recall bias, particularly for older children, as parents may have forgotten their children's exposure to the various HBV risk factors studied. By contrast, social desirability bias was minimized as participants' HBV status was not known at the time of the interviews, and therefore, it is unlikely that participants associated questions with HBV risk factors. Fourth, we did not use venous blood sampling, which is the reference method to detect $\mathrm{HBsAg}$. However, the choice to use DBS was based on WHO recommendations for areas where rapid diagnostic tests are unavailable or where there are no facilities or personnel to take venous blood samples. ${ }^{13}$ This method has a high performance with a $>90 \%$ diagnostic sensitivity and specificity compared with plasma or serum tests. Furthermore, we conducted a pilot study to determine HBsAg cut-off values (ie $1.0 \mathrm{lU} / \mathrm{ml}$ for negativity and $1.5 \mathrm{lU} / \mathrm{ml}$ for positivity) and all results within this range were systematically confirmed using blood samples. Of the 20 tests performed on children's blood samples in the AmBASS survey, only two were positive suggesting that we probably did not miss children with positive HBsAg. Moreover, available literature suggests that occult HBV infection is rare in children and it is therefore unlikely that occult HBV infection would change our results. ${ }^{15}$

The large sample size of our study population, and its consequent representativeness of children living in the Niakhar HDSS, are the major strengths of this work. Other strengths include the wide range of risk factors studied, as well as the measurement of the risk of horizontal transmission (thanks to almost all members of the same household being tested).

In conclusion, chronic HBV infection prevalence was low in children born in Senegal in the 11 years after the introduction of the hepatitis B vaccine, showing the success of the country's HBV vaccination program. Having an $\mathrm{HBsAg}$-positive sibling (same mother), home birth, lifetime stitches and tattoos were all risk factors associated with chronic HBV infection. Screening of high-risk groups-including pregnant women and siblings of HBsAg-positive individuals-is essential to further reduce chronic HBV infection prevalence in children in Senegal. Increasing awareness of infection control procedures and encouraging delivery in healthcare facilities are also essential interventions.

\section{1 | Statement of significance}

This is one of the few studies to date in SSA estimating the prevalence of chronic HBV infection and identifying associated risk factors in children born after the introduction of the HBV vaccine. Our results highlight the success of the Senegalese vaccination program. Strengthening hepatitis B screening of high-risk groups and promoting delivery in healthcare facilities are key interventions to reduce the hepatitis B burden in Senegal. Increasing awareness of hepatitis $B$ prevention and control measures is needed, both in Senegal and in other sub-Saharan African countries. 


\section{ACKNOWLEDGEMENTS}

We thank our partners at the National Viral Hepatitis Program and Ministry of Health and Social Action in Senegal, all the healthcare workers and individuals who agreed to participate in the study, as well as the field team: Modou Diome, Ndèye Selbé Diouf, Khadim Gueye, Moussa Gueye, Ibrahima Ndaw, Fadiène Ndiaye, Mayé Ndour, Coumba Sandiane Sène (interviewers), Khady Ba Gaye, Fatima Sène (nurses), Ngor Gousse Faye and Etienne Silmang Ndong (drivers). Our thanks also to the ANRS Emerging Infectious Diseases research agency for its financial and technical support, especially Nicolas Rouveau and Maria Camila Calvo Cortes. Finally, we thank Philippe Colson and Léa Luciani for the DBS quality control, and Jude Sweeney (Milan, Italy) for the English revision and copyediting of the manuscript.

\section{CONFLICT OF INTEREST}

The authors declare no conflicts of interest.

\section{AUTHORS' CONTRIBUTIONS}

$S B$ and $A D$ were the principal investigators of the ANRS 12356 AmBASS study and ensured its overall coordination. FM contributed to the design of the study protocol. $\mathrm{MC}$ and EB were in charge of the project management. $E B$ and $A D$ were responsible for the overall management of data collection. CS provided administrative, technical and material support for the study's implementation. GL and CTK were responsible for the analysis of biological data. LP provided support during data collection, performed the data analysis and wrote the first draft of the manuscript. FM, CP and SB provided support for the data analysis. All authors contributed to data interpretation, made critical comments on the draft manuscript and approved the final version of the manuscript for submission.

\section{DATA AVAILABILITY STATEMENT}

The data that support the findings of this study are available on request from the corresponding author. The data are not publicly available due to privacy or ethical restrictions.

\section{ORCID}

Lauren Périères (D) https://orcid.org/0000-0001-8895-5909 Camelia Protopopescu (D) https://orcid.org/0000-0003-0164-7917 Fabienne Marcellin (D) https://orcid.org/0000-0001-8853-3829 Marion Coste (D) https://orcid.org/0000-0001-9005-240X Aldiouma Diallo (D) https://orcid.org/0000-0003-0792-6722 Cheikh Sokhna (D) https://orcid.org/0000-0003-4810-8232 Sylvie Boyer (D) https://orcid.org/0000-0002-7567-5200

\section{REFERENCES}

1. WHO. Global Hepatitis Report 2017 [Internet]. World Health Organization; 2017 [cited 2020 Aug 10]. Available from: https:// www.who.int/hepatitis/publications/global-hepatitis-report2017/ en/

2. The Polaris Observatory Collaborators. Global prevalence, treatment, and prevention of hepatitis B virus infection in 2016: a modelling study. Lancet Gastroenterol Hepatol. 2018;3(6):383-403.
3. WHO. Hepatitis B vaccines: WHO position paper - July 2017. WHO Weekly epidemiological record. 2017;92(27):369-92.

4. Spearman CW, Afihene M, Ally R, et al. Hepatitis B in sub-Saharan Africa: strategies to achieve the 2030 elimination targets. Lancet Gastroenterol Hepatol. 2017;2(12):900-909.

5. WHO. Global health sector strategy on viral hepatitis 2016-2021 [Internet]. WHO; 2016 [cited 2020 Aug 10]. Available from: https:// www.who.int/hepatitis/strategy2016-2021/ghss-hep/en/

6. Pinho-Nascimento CA, Bratschi MW, Höfer R, et al. Transmission of hepatitis $B$ and $D$ viruses in an African rural community. mSystems. 2018;3(5).e00120-18.

7. Keane E, Funk AL, Shimakawa Y. Systematic review with metaanalysis: the risk of mother-to-child transmission of hepatitis $B$ virus infection in sub-Saharan Africa. Aliment Pharmacol Ther. 2016;44(10):1005-1017.

8. Dumpis U, Holmes EC, Mendy M, et al. Transmission of hepatitis B virus infection in Gambian families revealed by phylogenetic analysis. J Hepatol. 2001;35(1):99-104.

9. Howell J, Lemoine M, Thursz M. Prevention of materno-foetal transmission of hepatitis B in sub-Saharan Africa: the evidence, current practice and future challenges. J Viral Hepat. 2014;21(6):381-396.

10. Eke CB, Ogbodo SO, Ukoha OM, et al. Seroprevalence and risk factors of hepatitis $B$ virus infection among adolescents in Enugu, Nigeria. J Trop Pediatr. 2015;61(6):407-413.

11. Lingani $M$, Akita $T$, Ouoba $S$, et al. The changing epidemiology of hepatitis $B$ and $C$ infections in Nanoro, rural Burkina Faso: a random sampling survey. BMC Infect Dis. 2020;20(1):46.

12. McMahon BJ. The natural history of chronic hepatitis B virus infection. Hepatology. 2009;49(S5):S45-55.

13. WHO. Guidelines on hepatitis $B$ and $C$ testing [Internet]. WHO; 2017 [cited 2020 Aug 10]. Available from: https://www.who.int/ hepatitis/publications/guidelines-hepatitis-c-b-testing/en/

14. Shimakawa Y, Yan H-J, Tsuchiya N, Bottomley C, Hall AJ. Association of early age at establishment of chronic hepatitis B infection with persistent viral replication, liver cirrhosis and hepatocellular carcinoma: a systematic review. PLoS One. 2013;8(7):e69430.

15. Lo G, Sow-Sall A, Diop-Ndiaye H, et al. Hepatitis B virus (HBV) infection amongst children in Senegal: current prevalence and seroprotection level. Pan Afr Med J. 2019;32(140). https://doi. org/10.11604/pamj.2019.32.140.14485

16. Rey-Cuille M-A, Njouom R, Bekondi C, et al. Hepatitis B virus exposure during childhood in Cameroon, Central African Republic and Senegal after the integration of HBV vaccine in the expanded program on immunization. Pediatr Infect Dis J. 2013;32(10):1110-1115.

17. Argaw B. Sero-prevalence of hepatitis B virus markers and associated factors among children in Hawassa City, southern Ethiopia. BMC Infect Dis. 2020;20(1):528.

18. Ezeilo MC, Engwa GA, Iroha RI, Odimegwu DC. Seroprevalence and associated risk factors of hepatitis $B$ virus infection among children in Enugu metropolis. Virology (Auckl). 2018;9:1178122X1879285.

19. Coste $M$, De Sèze M, Diallo A, Carrieri MP, Marcellin F, Boyer S. Burden and impacts of chronic hepatitis $B$ infection in rural Senegal: study protocol of a cross-sectional survey in the area of Niakhar (AmBASS ANRS 12356). BMJ Open. 2019;9(7):e030211.

20. Delaunay V, Douillot L, Diallo A, et al. Profile: the Niakhar health and demographic surveillance system. Int J Epidemiol. 2013;42(4):1002-1011.

21. Lange B, Cohn J, Roberts T, et al. Diagnostic accuracy of serological diagnosis of hepatitis $C$ and $B$ using dried blood spot samples (DBS): two systematic reviews and meta-analyses. BMC Infect Dis. 2017;17(S1):700.

22. Mohamed S, Raimondo A, Pénaranda G, et al. Dried blood spot sampling for hepatitis B virus serology and molecular testing. PLoS One. 2013;8(4):e61077.

23. Hoffman JIE. Negative binomial distribution. In: Mica H, Rafael T, Mariana K, Julia H, Victoria P, eds. Biostatistics for Medical 
and Biomedical Practitioners. Cambridge, USA: Academic Press; 2015:279-284.

24. Szmuness W, Prince AM, Diebolt G, et al. The epidemiology of hepatitis $B$ infections in Africa: results of a pilot survey in the republic of Senegal. Am J Epidemiol. 1973;98(2):104-110.

25. Rey-Cuille M-A, Seck A, Njouom R, et al. Low immune response to hepatitis B vaccine among children in Dakar, Senegal. PLoS One. 2012;7(5):e38153.

26. Whittle $H$, Inskip $H$, Bradley $A K$, et al. The pattern of childhood hepatitis B infection in two Gambian villages. J Infect Dis. 1990;161(6):1112-1115.

27. Périères L, Marcellin F, Lo $G$, et al. Hepatitis $B$ vaccination in Senegalese children: coverage, timeliness, and sociodemographic determinants of non-adherence to immunisation schedules (ANRS 12356 AmBASS Survey). Vaccines. 2021;9(5):510.

28. Howell J, Pedrana A, Schroeder SE, et al. A global investment framework for the elimination of hepatitis B. J Hepatol. 2021;74(3):535-549.

29. Mauss S, Pol S, Buti M, et al. Late presentation of chronic viral hepatitis for medical care: a consensus definition. BMC Med. 2017;15(1):92.

\section{APPENDIX}

The ANRS 12356 AmBASS survey Study Group (ANRS Emerging Infectious Diseases; AmBASS: Burden and impacts of hepatitis B virus infection in rural Senegal)

Cyril Bérenger; Marwan al Qays Bousmah; Sylvie Boyer; Patrizia Carrieri; Marion Coste; MaëlleSeze; Tchadine Djaogol; Gwenaëlle Maradan; Fabienne Marcellin; Carole Treibich; Camelia Protopopescu (Aix Marseille Univ, INSERM, IRD, SESSTIM, Sciences Economiques \& Sociales de la Santé \& Traitement de l'Information Médicale, Marseille, France). El hadji Ba; Aldiouma Diallo; Fambaye Dièye; Assane Diouf; Elhadji Bilal Faye; Assane Ndiaye; Lauren Périères; Cheikh Sokhna; Mouhamadou Baba Sow (UMR VITROME, IRD-Université Aix Marseille, AP-HM, SSA, IHU-Méditerranée Infection, Marseille, France et Campus International IRD-UCAD de I'IRD, Dakar, Sénégal). Coumba Touré Kane; Gora Lo; Anna Julienne Selbé Ndiaye; Samba Ndiour (Institut de Recherche en Santé, de Surveillance Epidémiologique et de Formation, Dakar, Sénégal). Philippe Halfon; Sofiane Mohamed (Hôpital Européen, Marseille, France). Nicolas Rouveau; Maria-Camila Calvo Cortès; Gabrièle Laborde-Balen (ANRS Emerging Infectious Diseases, Paris, France). Martine Audibert, Fatou Fall, Ibrahima Gueye, Karine Lacombe, Moussa Seydi, Yusuke Shimakawa, Edouard Tuaillon, Muriel Vray (AmBASS scientific advisory board). 